\title{
Long period grating inscribed in multimode fibre interferometer and its application in refractive index sensing
}

\author{
Pengfei Wang*a, ${ }^{\mathrm{a}, \mathrm{b}}$, Haoyu Zhao ${ }^{\mathrm{b}}$, Gilberto Brambilla ${ }^{\mathrm{c}}$, Gerald Farrell ${ }^{\mathrm{a}}$, Libo Yuan ${ }^{\mathrm{b}}$ \\ ${ }^{a}$ Photonics Research Centre, Dublin Institute of Technology, Kevin Street, Dublin 8, Ireland; \\ ${ }^{b}$ Key laboratory of In-fiber Integrated Optics of Ministry of Education, College of Science, Harbin \\ Engineering University, Harbin 150001, China \\ 'Optoelectronics Research Centre, University of Southampton, Southampton SO17 1BJ, United \\ Kingdom
}

\begin{abstract}
A long period grating (LPG) is inscribed by a femtosecond laser in the multimode region of a singlemode-multimode singlemode fibre device to provide a compact refractive index sensor. An average sensitivity of $39 \mathrm{~nm} / \mathrm{RIU}$ and a resolvable index change of $2.56 \times 10^{-4}$ are obtained experimentally with a $44.4 \mathrm{~mm}$ long multimode fibre over a measured refractive index range of 1.33-1.35. Because of its compactness, ease of fabrication, linear response, high sensitivity, easy connectivity to other fiberized optical components and low cost, this refractometer could find various applications in chemical and biological sensing.
\end{abstract}

Keywords: Fibre optic sensor, modes interference, long period grating, femtosecond laser, refractometer

\section{INTRODUCTION}

Optical fibre based sensing devices offer numerous advantages over conventional electrical sensors due to their immunity to electromagnetic interference, , compact size, high sensitivity and capability of remote sensing. Optical fibre based refractive index (RI) sensors are promising and attractive in chemical and biotechnology applications for their superior performance. In the past few years, several types of refractive index optical fibre sensors have been developed. The most common approaches rely on fibre Bragg gratings (FBGs) [1,2], long period fibre gratings (LPFGs) [3,4], microbending [5], Fabry-Perot interferometers [6,7], and microfibre coil resonators [8]. However, most of them require expensive fibres or equipment to fabricate.

An inexpensive singlemode-multimode-singlemode (SMS) fibre based sensor utilizing multimode interference in the multimode fibre (MMF) core section has been proposed recently [9-12]. The SMS fibre based refractive index sensor reported in [12] allows for the measurement of the external refractive index with a maximum sensitivity of $1815 \mathrm{~nm} / \mathrm{RIU}$ (refractive index unit) and a dynamic range of 0.095 from 1.342 to 1.437 . However, a long portion of an MMF needs to be etched chemically and exposed to the surrounding medium in order to achieve a large refractive index change, resulting in the need to use expensive and dangerous chemical solutions, such as Hydrogen Fluoride (HF). For example, in order to obtain the maximum sensitivity of $1815 \mathrm{~nm} / \mathrm{RIU}$ presented in Ref. [12], experimentally the diameter of the MMF part needs to be etched down to $80 \mu \mathrm{m}$ and the length of the MMF needs to be controlled accurately to within a fraction of a mm, while the overall length of $42 \mathrm{~mm}$, is challenging in that there is in significant risk of breakage and a degree of difficulty in manipulating the fibre. Recently significant effort has been devoted to developing long period fibre grating devices in a range of applications such as optical communications, fibre lasers and fibre optics sensing systems. One approach to achieve the requirements is to employ an infrared femtosecond laser, which was first proposed by Konto et al in [13]. LPFGs fabricated by this technique showed a better aging stability combined with a lower temperature dependence, compared with those fabricated by ultraviolet (UV) lasers. Compared with SMS fibre structure based fibre sensors, LPFG based refractometers do not need an extra-chemical etching process and the cladding modes excited by the LPFG can directly sense the RI changes of the surrounding medium. To combine the advantages of both of the fibre sensing structures together, therefore An LPFG on multimode fibre based devices have been reported for a range of applications, such as for chemical sensing previously [14].

In order to further develop the LPFG into a SMS hybrid structure capable to perform a sensing application, in this paper an LPFG is directly written by a femtosecond laser in an MMF. In the MMF cladding section, the cladding modal *pengfei.wang@dit.ie; phone +353 1402 4831; fax +353 1402 4569; http://www.electronics.dit.ie/postgrads/pwang/ 
propagation excited by the LPFG depends on the external refractive index changes, therefore this proposed SMF-MMF with an LPFG-SMF (SMLS) structure can be used for an RI sensing. A femtosecond laser direct written method can be potentially used to fabricate a highly sensitive refractive index fibre optic sensor.

The schematic configuration of the SMLS fabricated in the experiments is shown in Fig. 1: the fibre structure under consideration consists of an input and an output SMF and a MMF with LPFG.

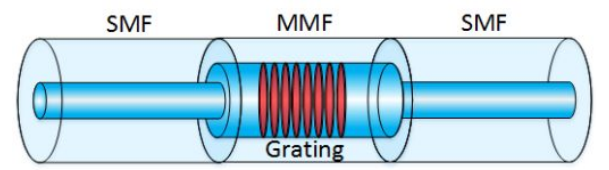

Figure 1. Schematic of the proposed SMF-MMF with LPFG-SMF (SMLS) structure.

\section{THEORETICAL ANALYSIS OF THE HYBRID FIBRE STRUCTURE}

First of all, in order to analyse light propagation differences between the traditional SMS and the SMLS structures, simulations based on the above hybrid fiber structures were carried out by using the well-known beam-propagation method. In the simulation, the singlemode fibre used, SMF 28, has core and cladding RIs of 1.4504 and 1.4447, respectively, with core and cladding diameters of $8.3 \mu \mathrm{m}$ and $125 \mu \mathrm{m}$, respectively; a multimode fiber AFS105/125Y is chosen with a NA of circa 0.22 and a fibre core and cladding diameters of $105 \mu \mathrm{m}$ and $125 \mu \mathrm{m}$, respectively. The MMF section has a length of $44 \mathrm{~mm}$. The grating pitch $\Lambda$ is $480 \mu \mathrm{m}$ and the period number is 80 . Figure 2(a) and (b) show light propagation along the SMS and SMLS structure, respectively, at the wavelength $1550 \mathrm{~nm}$. From Figure 2a, one can see that as light is injected from the input SMF to the MMF, multiple modes are excited, and multimode interference occurs within the MMF. When the LPFG is written on the MMF section, one can see that the output power of the SMF changes significantly. This indicates the LPFG written has a significant influence on the nature of the multimode interference in the MMF section.

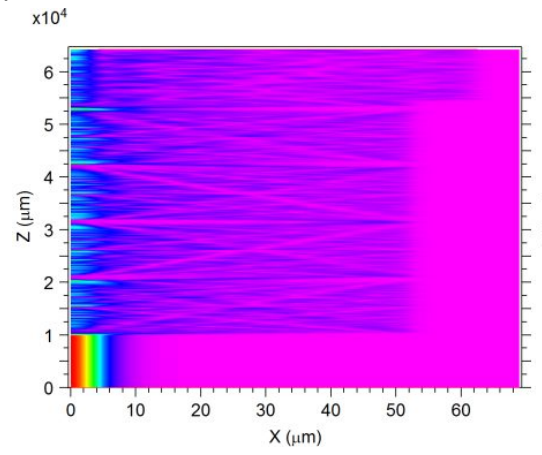

(a)

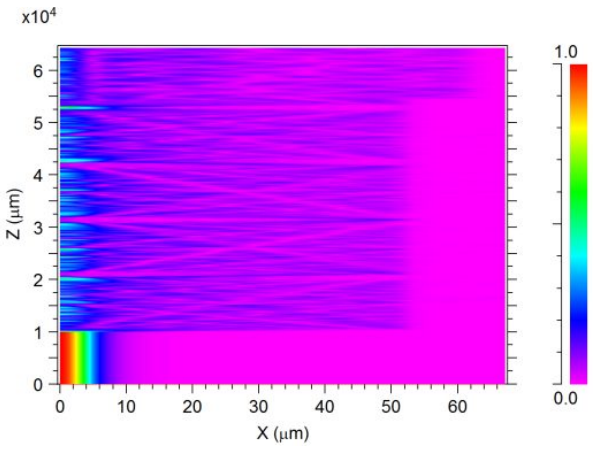

(b)

Figure 2. Calculated light propagation within the (a) SMS and (b) SMLS.

\section{FABRICATION OF SMLS STRUCTURE}

Figure 3 shows the schematic of the experimental setup used to fabricate the LPFG. An 800-nm laser beam (Ti: sapphire regenerative amplifier system with a pulse compressor; $120 \mathrm{fs}$; $1 \mathrm{kHz}$; single pulse energy up to $5 \mathrm{~mJ}$; beam diameter: $2.5 \mathrm{~mm}$ ) is focused onto a MMF by using an UV fused silica Plano-Convex cylindrical lens. The SMS fibre structure is mounted on two high-precision 3-axis translation stages and the position of the sandwiched MMF is precisely controlled with the help of a CCD camera. During the fabrication of the LPFG, the LPFG transmission spectrum was monitored in situ with a broadband light source and an optical spectrum analyzer (OSA). The laser beam with a pulse energy of circa $8 \mu \mathrm{J}$ was adopted for direct writing the LPFG. Periodic modifications can be produced by repeating the scanning process along the fiber length with identical spacing which is equal to the grating pitch $\Lambda$. The first sample we fabricated has a period of $450 \mu \mathrm{m}$ and the total length of this LPFG is $36 \mathrm{~mm}$.

The transmission spectra prior to writing the LPFG which is effectively that of the SMS and the of the SMLS over a wavelength range from $\lambda=1500 \mathrm{~nm}$ to $1600 \mathrm{~nm}$ are shown in Fig. 4. As expected, more interference dips appear over the 
wavelength range for the SMLS because of the presence of the LPFG, with the intensity of multimode interference in the MMF increasing correspondingly. The transmission dips show extinction ratios $>10 \mathrm{~dB}$.

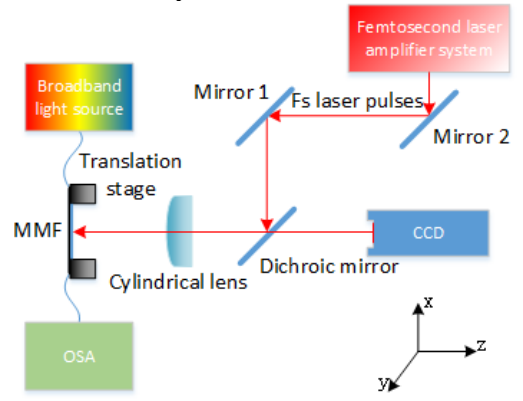

Figure 3. Schematic of experimental setup for fabricating the SMLS structure.

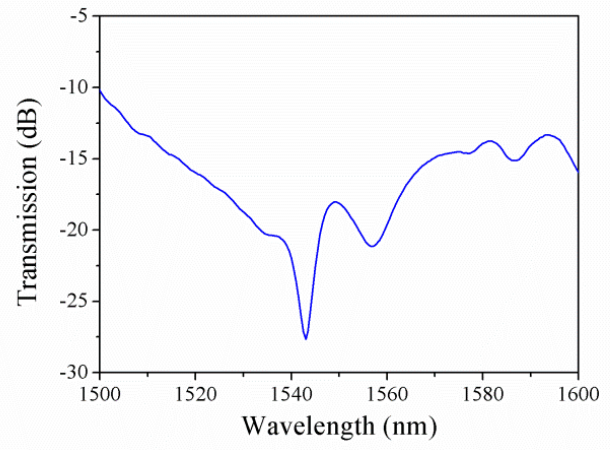

(a)

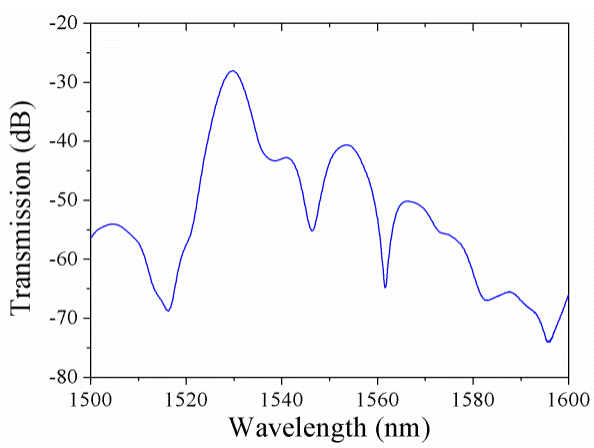

(b)

Figure 4. Transmission spectra of (a) SMS and (b) SMLS structures.

\section{MEASUREMENTS FOR REFRACTIVE INDEX SENSING}

The refractive index sensing measurement was performed at a room temperature $\left(\sim 20^{\circ} \mathrm{C}\right)$ with a series of calibrated RI liquids (1.33 1.35 with an interval of 0.005, RI error \pm 0.0002$)$. The SMLS structure was inserted into each RI liquid and transmission spectra were recorded by an optical spectrum analyzer (OSA).

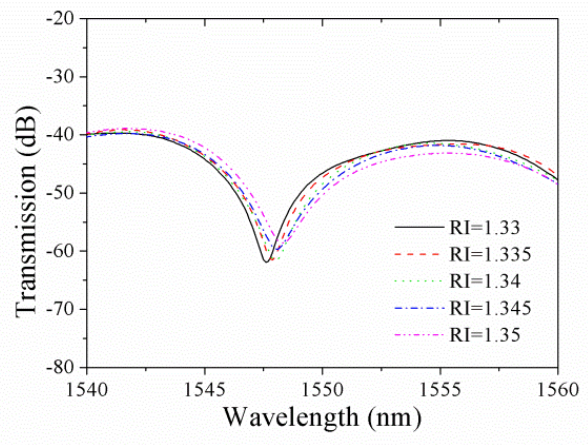

(a)

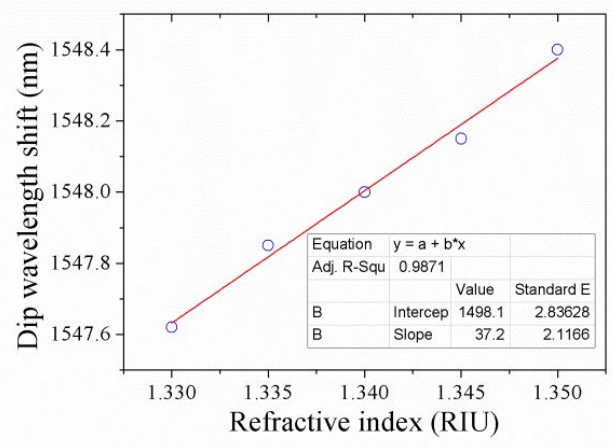

(b)

Figure. 5 (a) Spectral shift of the peak at $\sim 1548 \mathrm{~nm}$ for refractive index increasing from 1.33 to 1.35 ; (b) The wavelength shift of the peak as a function of RI.

Figure 5(a) shows the spectral shift of the dip at $\sim 1548 \mathrm{~nm}$ for refractive indices increasing from 1.33 to 1.35 with an interval of 0.005 . As the refractive index increases, the spectrum shows a redshift. The dip wavelength shift as a function of RI and the corresponding linear fit are presented in Figure 5(b). An average sensitivity of circa $39 \mathrm{~nm} / \mathrm{RIU}$ over an RI range of $1.33 \sim 1.35$ is achieved, resulting in a resolvable index change of $2.56 \times 10^{-4}$ for a resolvable wavelength change of $10 \mathrm{pm}$. 


\section{CONCLUSION}

A hybrid fibre refractometer based on an SMS with an LPFG fabricated by a femtosecond laser has been demonstrated. The refractometer has an average sensitivity of $39 \mathrm{~nm} / \mathrm{RIU}$ and a resolvable index change of $2.56 \times 10^{-4}$ for a resolvable wavelength change of $0.01 \mathrm{~nm}$. The sensitivity can be improved by either optimizing the parameters of the LPFG or the position of the LPFG on the MMF. This fibre sensor offers several advantages, including ease of fabrication, linear response, high sensitivity, ease of interconnection with other fiberized optical components and low cost. The device is a promising candidate for sensing in various chemical and biological applications.

\section{ACKNOWLEDGEMENTS}

GB gratefully acknowledges the Royal Society (London, UK) for his University Research Fellowship. This work was supported by the 111 project (B13015), at Harbin Engineering University. This publication has emanated from activity supported in part by Science Foundation Ireland (SFI) under the International Strategic Cooperation Award Grant Number SFI/13/ISCA/2845.

\section{REFERENCES}

[1] Han, M., Guo, F. W. and Lu, Y. F., "Optical fiber refractometer based on cladding-mode Bragg grating," Optics Letters 35, 399-401 (2010).

[2] Meng, H. Y., Shen, W., Zhang, G. B., Tan, C. H. and Huang, X. G., "Fiber Bragg grating-based fiber sensor for simultaneous measurement of refractive index and temperature," Sensors and Actuators B-Chemical 150, 226229 (2010).

[3] Li, J. L., Zhang, W. G., Gao, S. C., Geng, P. C., Xue, X. L., Bai, Z. Y. and Liang, H., "Long-Period Fiber Grating Cascaded to an S Fiber Taper for Simultaneous Measurement of Temperature and Refractive Index," IEEE Photonics Technology Letters, 25, 888-891 ( 2013).

[4] Han, Q., Lan, X. W., Huang, J., Kaur, A., Wei, T., Gao, Z. and Xiao, H., "Long-Period Grating Inscribed on Concatenated Double-Clad and Single-Clad Fiber for Simultaneous Measurement of Temperature and Refractive Index," IEEE Photonics Technology Letters 24, 1130-1132 (2012).

[5] Wang, P., Semenova, Y., Wu, Q., Farrell, G., Ti, Y. and Zheng, J., "Macrobending single-mode fiber-based refractometer," Applied Optics 48(31), 6044-6049 (2009).

[6] Frazao, O., Caldas, P., Santos, J. L., Marques, P. V. S., Turck, C., Lougnot, D. J. and Soppera, O., "Fabry-Perot refractometer based on an end-of-fiber polymer tip," Optics Letters 34, 2474-2476 (2009).

[7] Kou, J. L., Feng, J., Wang, Q. J., Xu, F. and Lu, Y. Q., "Microfiber-probe-based ultrasmall interferometric sensor," Optics Letters 35, 2308-2310 (2010).

[8] Xu, F. and Brambilla, G., "Demonstration of a refractometric sensor based on optical microfiber coil resonator," Applied Physics Letters 92, 101126 (2008).

[9] Wang, P., Brambilla, G., Ding, M., Semenova, Y., Q. Wu, G. Farrell, "A high sensitivity, evanescent field refractometric sensor based on tapered multimode fiber interference," Opt. Lett. 36(12), 2233-2235 (2011).

[10] Wang, P., Brambilla, G., Ding, M., Lee, T., Bo, L., Semenova, Y., Wu, Q. and Farrell, G., "An enhanced refractometer based on periodically tapered small core singlemode fiber," IEEE Sensors J. 13(1), 180-185 (2013).

[11] Wang, P., Brambilla, G., Ding, M., Semenova, Y., Wu, Q. and Farrell, G., "Investigation of singlemodemultimode-singlemode and singlemode-tapered multimode-singlemode fibre structures and their application for refractive index sensing," J. Opt. Soc. Am. B 28(5), 1180-1186 (2011).

[12] Wu, Q., Semenova, Y., Wang P. and Farrell, G., "High sensitivity SMS fiber structure based refractometer: analysis and experiment," Optics Express 19(9), 7937-7944 (2011).

[13] Kondo, Y., Nouchi, K., Mitsuyu, T., Watanabe, M., Kazansky, P. and Hirao, K., "Fabrication of long-period fiber gratings by focused irradiation of infrared femtosecond laser pulses," Opt. Lett. 24(10), 646-648 (1999).

[14] Lee, S. T., Kumar, R. D., Kumar, P. S., Radhakrishnan, P., Vallabhan, C. P. G. and Nampoori, V. P. N., "Long period gratings in multimode optical fibers: application in chemical sensing," Optics Communications 224, 237241 (2003). 